\title{
Double aortic arch in a 2 month old infant
}

\section{Summary}

We reported the case of a two-month-old infant with a diagnosis of double aortic arch, with persistence of the left ductus arteriosus, with a recurrent pneumonias, underwent surgery and after the diagnosis made by angiotomography, however retrospective, simple chest plate, esophagogram and bronchoscopy were compared where the same findings coincide in all of them; the patient was discharged for improvement.

Keywords: Double arch aortic, vascular rings, congenital vascular malformations
Volume II Issue 4 - 2018

Hernandez González Martha Alicia, De León Montes Alma Araceli, Gutierrez Luna Luis Javier, Gamboa Broca Brenda Veronica, Chavez Rodriguez Maria Silvia, Zavaleta Antunes Rodolfo

Department of radiology, University of Guanajuato, Mexico

Correspondence: De León Montes Alma Araceli, Department of radiology, University of Guanajuato, Mexico, Tel+ 013334935090,Email aracely.de.leon@hotmail.com

Received: June 13,2018 | Published: August 09, 2018

\section{Introduction}

The vascular rings are congenital malformations due to a defect in the development of the primitive aortic arches, these are anomalous vascular or ligamentous structures that surround the trachea and the esophagus, which are capable of producing symptoms by compression, the prevalence is difficult to estimate since many of them are asymptomatic, approximately $1 \%$ of all congenital cardiovascular anomalies are estimated.

\section{Case report}

Male infant of 2 months of age, product at term of the third gestation, obtained by iterative cesarean section, cries and breathes at birth, weighs $3.2 \mathrm{~kg}$, goes to a joint accommodation and is graduated binomial without complications, the mother mentions having attended with a normoevolutive pregnancy, going to prenatal control after the first month of pregnancy with a total of 10 to 12 consultations and 4 obstetric ultrasounds reported as normal, only folic acid intake from the first month.

The mother reports that at 5 days of extrauterine life the RN presents fatigue during the takings, with nasal congestion and respiratory rudeness, so he goes with his family doctor who refers him to normal, after 12 days he goes back to the office where they indicate washes nasal and frequent cleaning with knob, refer again without alterations.

After 2 months, he reported an increase in fatigue for the intake of intakes, but this time he presented perioral cyanosis and attack to the general state as well as increased respiratory difficulty on this occasion, denied fever, which is why he was taken to his clinic where he is admitted for probable pneumonia acquired in the community. Four days after admission, he underwent ventilatory phase III and transfer to the specialty unit, where a chest $\mathrm{x}$-ray is requested, which is assessed by a duty doctor, observing an endotracheal cannula with a tip to the proximal right main bronchus associated with atelectasis. is admitted to the intensive care service. He remains in therapy for 13 days, with a torpid evolution, however, he manages to keep phase I ventilation, so he is sent to the floor, to start orally, in a continuous floor with larynx stridor and respiratory rudeness, so it is decided to perform a series gastroduodenal esophagus, where extrinsic compression was reported at the junction of the upper third with the middle third of the esophagus, reported bronchoscopy with extrinsic compression apparently of pulsatile mass, angiotomography is requested with double aortic arch diagnostic printing with ring communication which conditions tracheal and esophageal compression. ${ }^{2}$ It is presented to the pediatric surgery service and is operated on, surgery without complications was reported with right posterior and left anterior aortic arch and persistence of the $3 \mathrm{~mm}$ ductus arteriosus. Goes to the apartment to continue driving, He is released to his home for improvement (Figures 1-4).
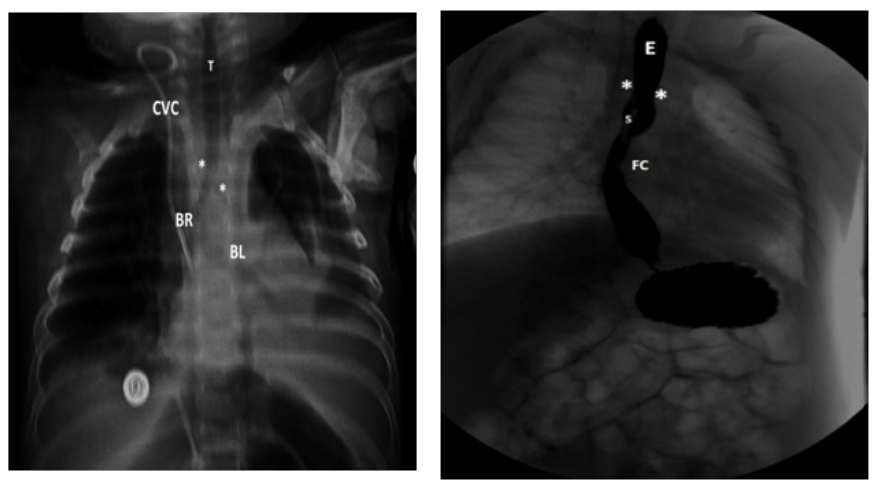

Figure I (A) simple chest PA plates, showing slight deviation of the trachea (T) to the left with right $\left(^{*}\right)$ and left $\left(^{*}\right)$ indentation before the bifurcation of the right main bronchus (BR) and left (BL). Radiopaque artifact in relation to central venous catheter (CVC) with distal tip in right atrium $B$ ) is corroborated with indentation esophagogram $(*)$ just at the same level observed in the simple plaque, but at the level of the esophagus (E) which is occupied by orogastric probe. Observing physiological stricture (FC) above the diaphragm. 

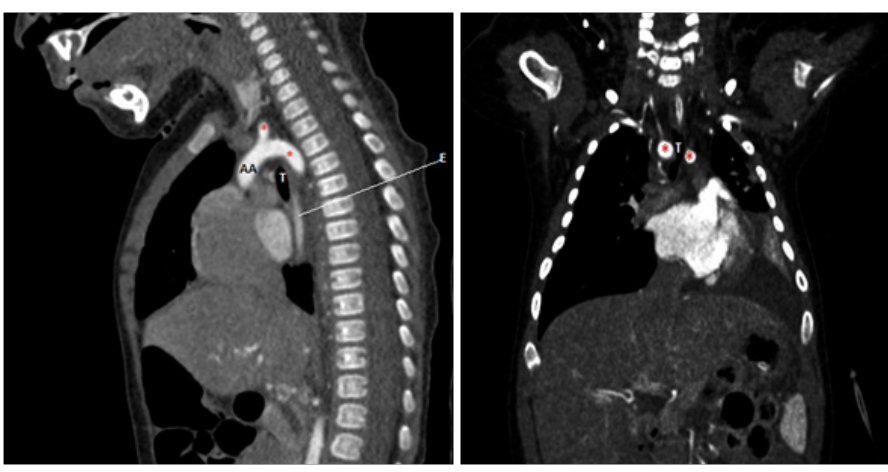

Figure 2 (A) Sagittal reconstruction of angiotomography showing the origin of the ascending aorta (AA) with two branches one right and one left in relation to the tracheal ring $(*)$ B) Coronal reconstruction demonstrates the decrease in the caliber of the trachea $(\mathrm{T})$ with right and left indentation prior to the bifurcation of the main bronchi.
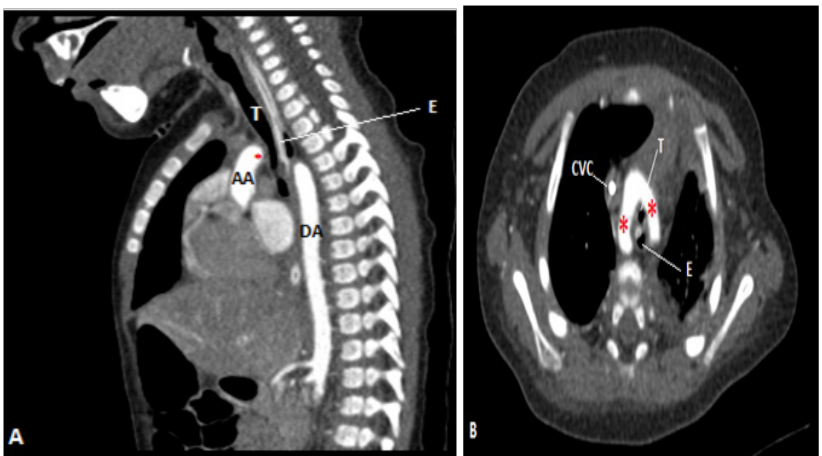

Figure 3 (A) sagital reconstruction of angiotomography in a lower cut, where the origin of the ascending aorta (AA) and the beginning of one of the branches that make up the vascular ring $\left(^{*}\right)$ can be seen. Note the decrease in the caliber of the trachea $(T)$ and the esophagus $(E)$ which is occupied by an orogastric tube, with a descending aorta (DA) of trajectory and usual caliber. B) axial cut of angiotomography where the vascular ring is clearly evident $(*)$, conditioning compression of trachea $(\mathrm{T})$ and esophagus (E) observing hyperdense material in relation to central venous catheter (CVC).
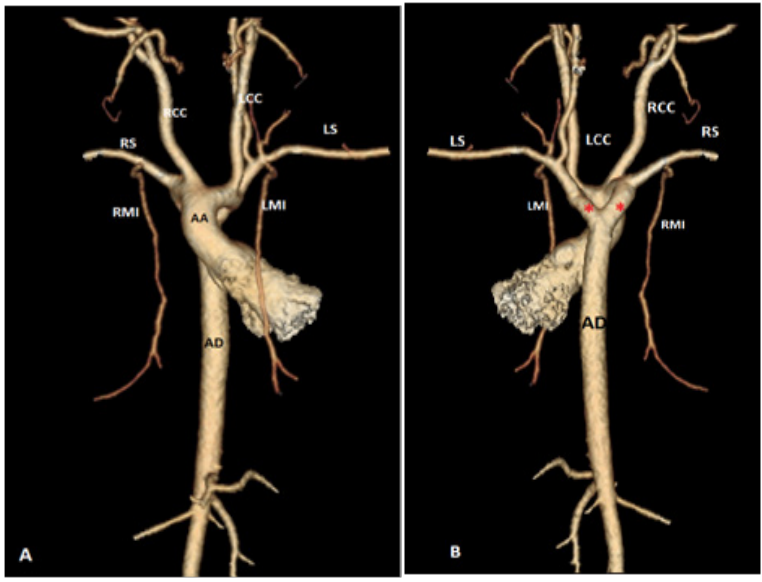

Figure $43 \mathrm{D}$ reconstruction of angiotomography A) anterior where emergence of ascending aorta (AA) is observed giving rise to the vascular ring and in turn each of them giving rise to the right common carotid artery (RCC) left common carotid artery (LCC), right subclavian artery (LR) and left subclavian artery (LS) respectively, with usual origin of right internal mammary artery (IMR) and left internal mammary artery (LMI) B) in posterior reconstruction the entire vascular ring is observed, with right dominance, descending aorta (AD) and usual caliber.

\section{Discussion}

Of the more than 100 cardiac and vascular morphological alterations, 6 are the most frequently observed, among them: anomalous left subclavian artery with left aortic arch, right aortic arch with left ligament, right aortic arch with aberrant left subclavian artery, compression tracheal through the in nominate artery, double aortic arch and sling of the pulmonary artery. ${ }^{3}$

The normal pattern is a left aortic arch, with a left descending thoracic aorta and a left duct or ligament extending from the proximal descending thoracic aorta to the left pulmonary artery. The branches of the arch are the right brachiocephalic artery (which divides into the common carotid and right subclavian artery), the left common carotid artery and the left subclavian artery. ${ }^{2}$

In the double aortic arch, the ascending aorta is divided into two arcs, right and left, that surrounds the trachea and esophagus and joins together behind the esophagus to form the descending aorta. From each of them, the carotid arteries are born independently from the front and the subclavian arteries from behind. Usually the left ductus persists, which when closed becomes a ligament. ${ }^{4}$

They can be identified through different image studies. In the chest $\mathrm{X}$-ray AP we can identify indentation of the tracheal air column, which will appear according to the side where the arc is present, in a double arc bilaterality is observed. In the case of our patient, the stenosis was observed before the bifurcation of the bronchi, with indentation in the right and just below this other on the left side. The esophagogram is also used for the diagnosis where we can see decrease in caliber at the level of the esophagus, either with indentation to the right, to the left or bilaterally depending on the alteration, the barium study is usually an indication for patients with data of recurrent gastroesophageal reflux associated with recurrent pneumonias, as was the case in our patient, with extrinsic compression, with a high suspicion of vascular ring, being a finding. Bronchoscopy is performed in patients without a clear diagnosis and to exclude other causes of respiratory distress in children, such as foreign bodies and subglottic stenosis, evaluation of complete tracheal rings and other congenital anomalies of the respiratory tract.

Echocardiography has a limited role in the evaluation of vascular rings due to the small field of vision. Magnetic resonance is one of the modalities used, with the advantage that it does not need iodinated contrast medium, it includes a wide field of vision, multiplanar images and adequate spatial resolution, however it is expensive, for some not very accessible, prolonged time and analgesic sedation are needed in young children. The angiotomography has turned out to be an image examination suitable for the diagnosis and characterization of the vascular rings, thus helping in surgical planning, including rapid acquisition time, high resolution, large field of vision, 3D reconstruction capacity, as well as evaluation of the rest of the organs and adjacent structures, without the need for analgesia, its main disadvantage is the use of contrast media and ionizing radiation. 2 However, it is currently possible to take measures in this regard, using a lower dose of radiation, as well as an adequate collimation, with a minimum dose for the administration of the contrast medium calculated per kilogram of weight, as was the case with our patient. I study the definitive one for the diagnosis and opportune surgery.

\section{Conclusion}

In recent years radiology has taken an important significance for the diagnosis of many pathologies, from the simple plate, which yields many data of various alterations with which we can reach a 
timely and accurate diagnosis, however it is important that the doctor First contact is aware of certain radiographic signs associated with the patient's clinic and thus avoid invasive studies and prolonged instances in hospitals, in this case the simple chest plate revealed stenosis at the tracheal level, with slight deviation from it towards the right. Although the vascular ring is not a frequent pathology, it is important to consider it in the face of a torpid evolution of the infant, with recurrent respiratory disease, weight or height that is low for age, and it can be complemented with a esophagram which shows a coincidence with the data found by simple plaque, or it could be requested in case of high suspicion from the doctor of first contact the angiotomography, thus avoiding delay in the diagnosis. As already mentioned, angiotomography has the disadvantage of requiring radiation and contrast medium for its realization, however it shows a high specificity for the diagnosis and surgical planning, therefore the doctor must be familiar with the findings by simple plate to avoid exposures unnecessary.

\section{Acknowledgments}

None.

\section{Conflict of interest}

The author declares that there is no conflict of interest.

\section{References}

1. Robert A Ordoñez-Ortega, Baena G, Ramirez J. Doble arco aórtico. Reporte y análisis de caso de un paciente colombiano, Rev Fac Med. 2015;63(4):733-738.

2. Etesami M, Ashwath R, Kanne J, et al. Computed tomography in the evaluation of vascular rings and slings. Insights Imaging; 2014;5(4):507-521.

3. Backer C, Mavroudis C. Vascular rings and pulmonary artery sling. In Pediatric Cardiac Surgery. 4h $^{\text {th }}$ Ed. Mavroudis C, Backer CL, editors. USA: Blackwell publishers; 2013.

4. Luis García-Guereta, Estefanía García-Cerro, Montserrat Bret-Zurita. 2016 Tomografía computarizada con multidetectores en las anomalías congénitas del arco aórtico: anillos vasculares. Rev Esp Cardiol. 2016;69(7):681-693. 